\title{
Polyurethane Resins as Resist Materials for Excimer Ablation Lithography(EAL)
}

\author{
Nobuaki Hayashi, Kenkichi Suzuki, Masaaki Matsuda, \\ Toshio Ogino, and Yoshifumi Tomita \\ Image-Related Device Development Centert, Electron Tube \& Devices Division, \\ Hitach Ltd., Kokubunji, Tokyo 185 Japan
}

\begin{abstract}
The excimer laser ablation lithography(EAL) is a process of direct patterning and developing of a resist film by photo-decomposition ablation. Taking advantage of EAL process, we have tried to apply this process to LCD fabrication. As the most fundamental problem for resist materials is the ablation rate, we have examined to measure the ablation rates of many kinds of polymers. Among them the most promising is the polyurethane resin (PU) which is synthesized from toluenediisocyanate and poly(ethylene glycol) derivatives in chlorobenzene solution. The ablation rate at $100 \mathrm{~mJ} / \mathrm{cm}^{2}$ fluence of $248 \mathrm{~nm}$ is more than $0.05 \mu \mathrm{m} / \mathrm{shot}$, which is the highest value of all the materials that we have examined. Through the investigations of structures of PU, we could elucidate the mechanisms of the high ablation rate, and accordingly the molecular design concept of the ablation resist.

Keywords:excimer laser, ablation, lithography, LCD, polyurethane
\end{abstract}

\section{Introduction}

After the pioneering work ${ }^{1}$ of Dr. Srinivasan in the early time of 1980 's, laser ablation of polymers has been intensively studied. Many papers were reported toward the application for high resolution lithography and dry etching process to make fine patterns on the silicon wafers. Recently, the excimer laser ablation phenomenon has been mainly applied to the micromachining of the polyimide layers on the print circuit boards. Accordingly the photodecomposition behaviors have been most intensively studied for the size of tens of micron of the thick polyimide films. However the ablation patterning is principally applicable thin resist film, in which case both the exposure and development processes are merged into one step. The advantages of this new Excimer Ablation Lithography(we call it EAL) is utterly obvious, and we have tried to apply EAL to LCD fabrication ${ }^{2}$. EAL actually consists of various kinds of technologies as is the case of the conventional photolithography, and here we confine ourselves to the new resist materials, that is, the ablation resist polymers which play the important role in EAL.

In this paper, we will discuss the polyurethane resins as a resist materials for EAL.

\section{Experimental}

\subsection{Materials}

Photoresists, commercially available polymers, reagents and organic solvents are used as received. PU resin from toluenediisocyanate (TDI) and polyethyleneglycol (PEG) was synthesized as follows. 5.8g of PEG (Mw 200) was dissolved in $100 \mathrm{ml}$ of chlorobenzene and $30 \mathrm{ml}$ of dichlorobenzene. The solution was stirred at $60^{\circ} \mathrm{C}$ under nitrogen atmosphere. $5.0 \mathrm{~g}$ of TDI in $50 \mathrm{ml}$ of chlorobenzene was added in a few minuets and then heated at $130^{\circ} \mathrm{C}$ for 2 hours. After standing overnight, the polymer was separated, collected and then dissolved in $100 \mathrm{ml}$ of $\mathrm{N}, \mathrm{N}$-dimethylacetoamide (DMA).

\subsection{Instruments}

UV absorption spectra were measured with a Hitachi U-3410 spectrometer. Infrared spectra were recorded on a Perkin-Elmer 1720 FTIR spectrophotometer using thin films spin coated on 
aluminum-deposited silicon wafer.

Irradiation have been carried out using an excimer laser(Lambda Physik LPX210i; KrF, $600 \mathrm{~mJ}$ output, max.) operated at a wavelength of $248 \mathrm{~nm}$, with a repletion rate of $100 \mathrm{~Hz}$, and a pulse duration of $30 \mathrm{~ns}$.

\section{Results and Discussion}

In the purpose to develop new resist for EAL, we have evaluated the many kinds of polymers. We sought the new resist materials specified following restrictions.

1. High ablation rate at $248 \mathrm{~nm}$ irradiation of $100 \mathrm{~mJ} / \mathrm{cm}^{2}$ fluence.

2. High resolution with debris free.

3 Well resistant to wet and dry etching.

Many kinds of polymers were examined as materials to be used under these restrictions. The resolution of the resist is determined by the photodecomposition center density and it is completely similar to the conventional photoresist. As the most fundamental problem is the ablation rate, we have examined to measure the ablated depth per pulse at $100 \mathrm{~mJ} / \mathrm{cm}^{2}$ fluence. The fluence of light was limited to avoid the damage of substrate. Results are shown in Table 1. As can be

Table 1 Ablation rates and absorption coefficients of polymer films

\begin{tabular}{|c|c|c|}
\hline Materials & $\begin{array}{c}\text { absorption } \\
\text { coefficient } \\
\left(110^{4} \mathrm{~cm}^{-1}\right)\end{array}$ & $\begin{array}{c}\text { ablation rate } \\
\text { ( } \mu \mathrm{m} / \text { shot) }\end{array}$ \\
\hline \multicolumn{3}{|l|}{ photoresist for g-line } \\
\hline $\begin{array}{c}\text { OFPR-800 } \\
\text { (Novolac resin \& NQD) }\end{array}$ & $4-5$ & $0.03-0.05$ \\
\hline Novolac resin & - & no ablation \\
\hline OFPR-800+NQD & 8 & 0.04 \\
\hline OFPR-800+pyrene & 10 & 0.04 \\
\hline cyclized rubber+azide & - & no ablation \\
\hline \multicolumn{3}{|l|}{ Electron beam resists } \\
\hline PMMA & 0.014 & no ablation \\
\hline PMMA+pyrene & 1.84 & no ablation \\
\hline poly( $\alpha$-methylstyrene) & - & 0.028 \\
\hline poly(methylpropenylketone) & 0.66 & no ablation \\
\hline \multicolumn{3}{|l|}{ Polyimides } \\
\hline PIQ-6200(Hitachi Chemical) & $12-15$ & 0.05 \\
\hline photosensitive polyimide & $8-10$ & 0.05 \\
\hline UR3100(Toray) & $2-3$ & 0.02 \\
\hline \multicolumn{3}{|l|}{ Polyurethanes } \\
\hline Isomelt1500(Schenectady) & $5-8$ & 0.05 \\
\hline Urea-A(Hitachi Chemical) & 0.2 & no ablation \\
\hline
\end{tabular}

seen in the Table 1, electron beam resists dose not ablate, and conventional photoresists for i-line or g-line show the ablation rate of about $0.03 \mu \mathrm{m} / \mathrm{shot}$. Polyimide resins show higher ablation rate than that of conventional photoresists. Photosensitive polyimide resists also show high ablation rate. After irradiation of polyimide films, there remains a residual materials on the substrate and the unexposed area. Among these evaluation, most promising is the PU resin.

To improve the ablation rate, we consider the structure of PU resins. As PU was synthesized from diisocyanate and diol, we have examined to use aromatic diisocyanate or aliphatic one. Chemical structure of these PUs are shown in Fig. 1. UV spectra of phenylcabamic acid ethyl ester (structure I) and carbamic acid phenyl ester (Structure II) were shown in Fig 2. The molar absorption coefficient at $248 \mathrm{~nm}$ are 6000 and 180 , respectively. Considering the ability of UV absorption, PUs from aromatic diisocyanates will be able to show higher ablation rate than that of aliphatic one.

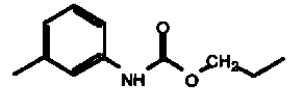

Structure I

Phenylcarbamic acid ester (phenyl carbamate)

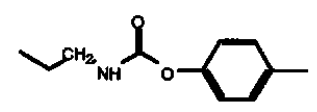

Structure II

Carbamic acid phenyl ester (carbamate phenyl ester)
Fig. 1 Structure of phenylcarbamate and carbamate phenyl ester

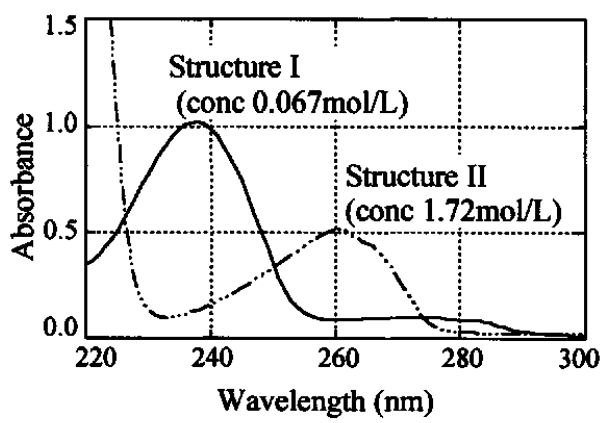

Fig. 2 UV spectra of carbamic acid ester

We have prepared the PU resin which is synthesized from toluenediisocyanate (TDI) and poly(ethylene glycol) (PEG) in chlorobenzene and dichloro-benzene. Reaction scheme is shown in Fig. 3. IR spectrum of the PU resin is shown in Fig. 4. As can be seen in Fig. 4, carbonyl band from amide structure is observed at $1730 \mathrm{~cm}^{-1}$ and isocyanate band at $2270 \mathrm{~cm}^{-1}$ is completely disappeared. The ablation rate of this PU film at 


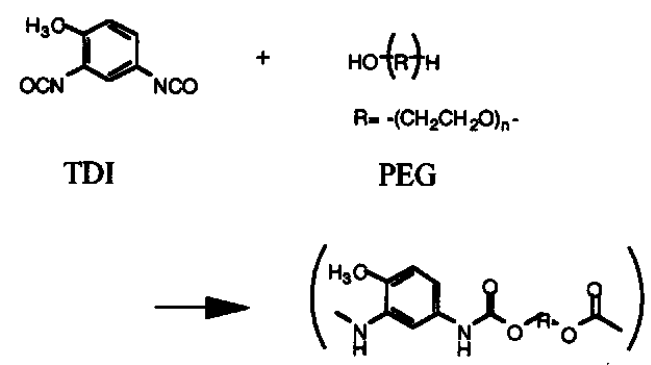

Polyurethane resin

Fig. 3 Reaction scheme of polyurethane resin

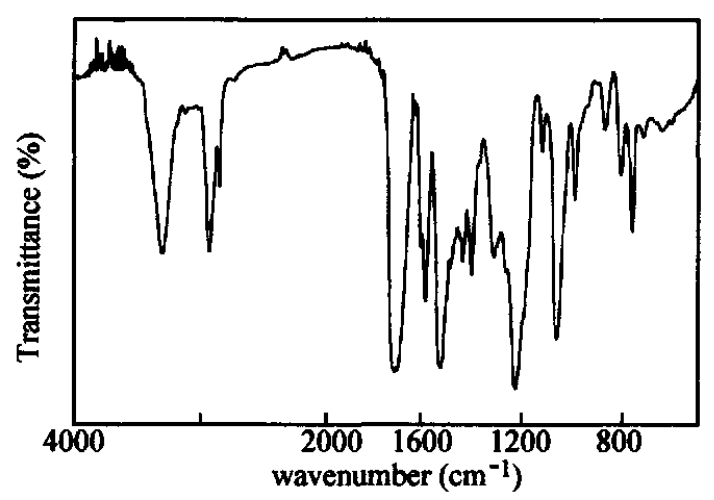

Fig. 4 IR spectra of polyurethane resin

$100 \mathrm{~mJ} / \mathrm{cm}^{2}$ fluence of $248 \mathrm{~nm}$ UV light is more than $0.05 \mu \mathrm{m} / \mathrm{shot}$, which is the highest value of all the materials that we have examined. Results are shown in Fig. 5. The total ablated depth increases linearly with the number of pulses delivered.

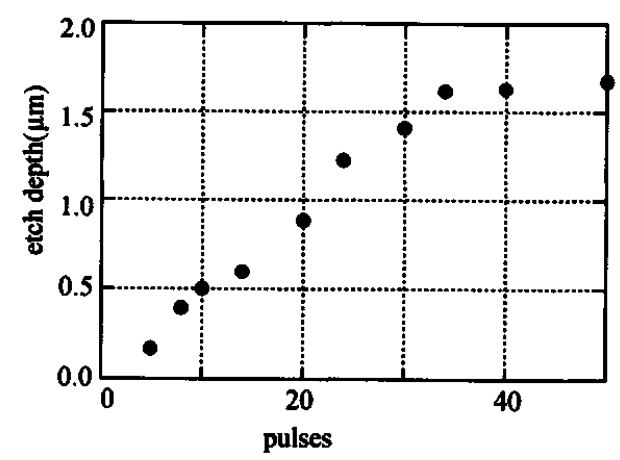

Fig. 5 Ablation rate of polyurethane resin

To understand the relationship between the absorbance of resist film and ablation rate, we have examined the ablation characteristics of PU resin film that have been physically doped with dye. The ablation rates as a function of mixing ratio with Disperse Red 1(DR1), which is famous dye using in nonlinear optical materials, to PU resin are examined. Results are shown in Fig. 6. As seen in Fig. 6, the ablation rates of resist film are not markedly dependent upon the ratio of DR1 it contain. Concequently, we have examined the ablation rate of PU which has DR1 structure at the end of polymer chain. The results are shown in Fig. 7. DR1-terminated PU shows almost same ablation rate. A same behavior among PU film, dye- doped film, and dye-terminated film can be presumed that photochemical or photothermal bond scission of PU chain caused the ablation of resist film.

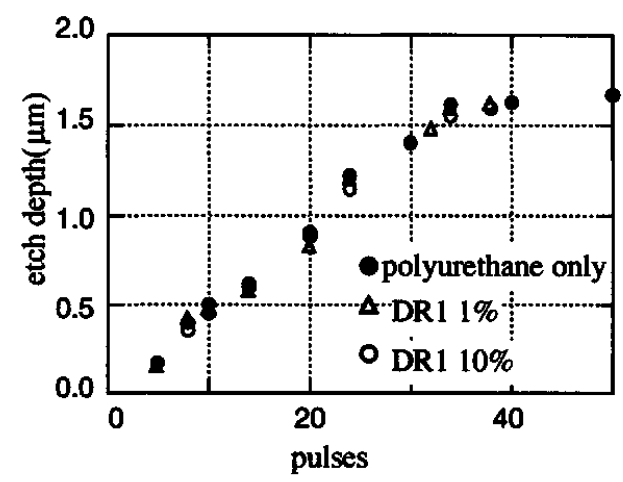

Fig. 6 Ablation rates of polyurethane resin doped with DR1
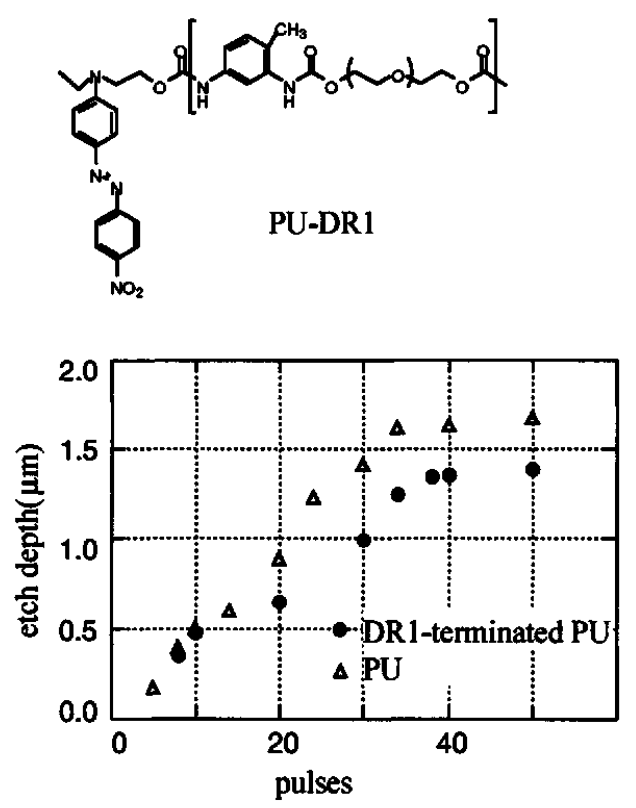

Fig. 7 Ablation rates of DR1-terminated PU 


\section{Conclusion}

We have examined many kinds of polymers as resist materials for excimer laser ablation lithography (EAL). In these evaluation, we have found that PU resin synthesized from toluenediisocyanate and polyethyleneglycol have shown high ablation rate without debris. The ablation rate at $100 \mathrm{~mJ} / \mathrm{cm}^{2}$ fluence of $248 \mathrm{~nm}$ is more than 0.05 $\mu \mathrm{m} / \mathrm{shot}$.

\section{References}

1. R. Srinivasan and V. Mayne-Banton, Appl. Phys. Lett. 41576 (1982); J. E. Andrew, P. E. Dyer, D. Forster and P. H. Key, Appl. Phys. Lett. 43717 (1983); T. F. Deutsch and M. W. Geis, J. Appl. Phys. 547201 (1983)

2. K. Suzuki, M. Matsuda, T. Ogino, N. Hayashi, T. Terabayashi and K. Amemiya, SPIE Proceedings 2992, 98 (1997) 